\title{
免疫球蛋白联合甲泼尼龙治疗急性格林巴利综合征的 效果分析
}

\author{
李明阳* \\ 商丘市第一人民医院急诊科，河南 476100
}

摘 要: 目的：研究探讨免疫球蛋白联合甲波尼龙治疗急性格林巴利综合征的临床效果。方法：选取我院2018年 2月 2019年2月收治的90例急性格林巴利综合征患者为研究对象, 将其分为两组, 对照组采取单纯免疫球蛋白治疗, 观察组采取免疫球蛋白联合甲波尼龙治疗，观察两组临床疗效。结果：观察组治疗总有效率、起效时间、住院时间以 及治疗费用等各项治疗指标均优于对照组 $(P<0.05)$; 治疗后观察组生活能力评分显著高于对照组 $(P<0.05)$; 观 察组与对照组不良反应发生率分别为 $4.44 \%$ 与 $6.67 \%(P>0.05)$ 。结论：给予急性格林巴利综合征患者免疫球蛋白联 合甲泼尼龙治疗可获得良好临床疗效，具有较高的安全性，在临床上具有推广应用价值。

关键词：免疫球蛋白；甲泼尼龙；急性格林巴利综合征

\section{Effect Analysis of Immunoglobulin Combined with Methylprednisolone in the Treatment of Acute Guillain Barre Syndrome}

\author{
Ming-Yang $\mathrm{Li}^{\star}$ \\ Emergency Department of Shangqiu First people’s Hospital, Shangqiu 476100, Henan, China
}

\begin{abstract}
Objective: To study the clinical effect of immunoglobulin combined with methylprednisolone in the treatment of acute Guillain Barre syndrome. Method: 90 patients with acute limbal syndrome in our hospital from February 2018 to February 2019 were selected and divided into two groups. The control group was treated with simple immunoglobulin, while the observation group was treated with immunoglobulin combined with methylprednisolone. Result: The total effective rate, onset time, hospitalization time and treatment cost of the observation group were better than those of the control group $(P<$ 0.05); after treatment, the life ability score of the observation group was significantly higher than that of the control group $(P<$ $0.05)$; the incidence of adverse reactions in the observation group and the control group were $4.44 \%$ and $6.67 \%$, respectively $(P$ $>0.05$ ). Conclusion: Immunoglobulin combined with methylprednisolone in the treatment of patients with acute Guillain Barre syndrome can obtain good clinical efficacy, with high safety, and has the value of clinical application.
\end{abstract}

Keywords: Immunoglobulin; methylprednisolone; acute Guillain Barre syndrome

\section{一、前言}

急性格林巴利综合征为一种自身免疫性疾病, 临床症状为反射功能障碍、感觉障碍、躯干及四肢肌肉痽瘾等, 对患 者日常生活有着非常严重的影响, 必须给予积极治疗 ${ }^{[1-2]}$ 。就临床研究来看, 吐过急性格林巴利综合征患者未及时采取治 疗干预, 病情持续发展将会损伤患者的脑部神经, 甚至会导致死亡。目前临床上以采用激素、免疫球蛋白等为主要治疗 方法, 根据研究结果显示, 联合免疫球蛋白与激素可以进一步提高临床疗效。根据我院所收治急性格林巴氏综合征患者 的临床用药情况，研究分析了免疫球蛋白联合甲泼尼龙治疗急性格林巴氏综合征的临床效果。详细报告如下。

\section{二、资料与方法}

(一) 一般资料

*通讯作者：李明阳，1983年2月，男，汉族，河南商丘人，现任商丘市第一人民医院急诊科主治医师，本科。研 究方向：神经病学，脑血管病及神经变性疾病。 
以我院收治的 90 例急性格林巴利综合征患者为研究对象, 对其临床资料做回顾性分析。根据采取治疗方案的不 同, 将其分为对照组和观察组, 每组有45例患者, 两组患者一般资料比较无差异 $(P>0.05)$ 。其中, 对照组中男 性和女性患者各有 23 例与 22 例, 最小年龄 20 , 最大 63 岁, 平均 $(41.2 \pm 5.6)$ 岁, 病程3 $46 \mathrm{~h}$, 平均病程 $(35.2 \pm 11.3)$ $\mathrm{h}$; 观察组中男性与女性患者各有 21 例和 24 例, 最小年龄 22 , 最大 64 岁, 平均 $(41.5 \pm 5.1)$ 岁, 病程 $3 \sim 44 \mathrm{~h}$, 平均病程 (35.1 \pm 10.9$) h$ 。本研究均经过患者知情同意，且通过医院伦理委员会批准。

\section{(二) 方法}

所有患者人院后均给予常规对症治疗, 对照组在常规治疗基础上, 对患者进行单纯的免疫球蛋白治疗, 即给药免 疫球蛋白 (华兰生物工程股份有限公司, 国药准字 $\mathrm{S} 10970032$ ) $0.4 \mathrm{~kg} / \mathrm{kg}$ 静滴, 1 次 $/ \mathrm{d}$, 持续治疗 $5 \mathrm{~d}$ 。同时, 需要给予患 者营养支持和抗感染治疗, 有效维持水电解质平衡, 预防应激性溃疡等症状的发生, 一旦发现需要及时给予治疗 ${ }^{[3-4]}$ 。 并且，对于病情严重的呼吸麻㿎患者，需要将气道切开借助呼吸机进行辅助治疗。

基于对照组给药方案, 观察组增加给药甲波尼龙 (天津金耀药业有限公司, 国药准字H20103047 ) 15 20 mg 加人 $5 \%$ 葡萄糖注射液150 250 mL中静滴, 1 次/ d, 持续治疗 $5 \mathrm{~d}$ 。整个治疗过程中, 要密切关注患者水电解质情况, 确保水 电解质平衡。医护人员还需要根据患者病情提前制定好应激性反应预防对策 ${ }^{[5-6]}$ 。

参数设置: 应用同步间歇指令进行通气, 呼吸频率调整为 $14 \sim 8$ 次 $/ \mathrm{min}$, 呼吸比例设定为 $1: 2$, 潮气量为 $8 \mathrm{~mL} / \mathrm{kg}$, 吸 氧浓度尽量不要超过 $55 \%$, 通气压力 $16 \mathrm{~cm} \mathrm{H}_{2} \mathrm{O}$, 流速 $40 \mathrm{~L} / \mathrm{min}$, 并且要及时根据患者血气分析结果来调整呼吸机参数 ${ }^{[7]}$ 。

\section{（三）观察指标与评价标准}

观察比较两组用药起效时间 (治疗后病情明显好转, 说话声音小、四肢肌无力等临床症状好转, 生活自理能力提 高) 与住院时间。应用日常生活活动能力评定 (ADL) 量表对两组患者治疗前后的生活能力进行评价, 分数越高则生 活能力越强。另外, 观察比较两组治疗后不良反应发生情况, 主要为恶心、头痛、发热、面部潮红等 ${ }^{[8-9]}$ 。

评价比较两组治疗有效率, 分为显效、有效与无效三个维度。显效即患者治疗后说话声音小、呼吸困难、四肢肌肉 无力等症状全部消失; 有效即患者治疗后各临床症状得到一定好转, 四肢肌力 I III级, 生活自理能力方面存在一定限 制; 无效即患者治疗后各临床症状无明显改善, 生活自理能力较治疗前无变化 ${ }^{[10-11]}$ 。治疗总有效率 $=$ 显效率+有效率。

(四) 统计学方法

文章数据用SPSS19.0软件, 计量资料采用 $\mathrm{T}$ 检验, 计数资料以 $\chi 2$ 检验。若 $P<0.05$, 则有统计学意义。

\section{三、结果}

（一）比较两组治疗总有效率

观察组与对照组治疗总有效率分别为 $95.56 \%$ 和 $82.22 \%$, 观察组显著优于对照组, 差异具有统计学意义 $(P<$ $0.05)$ ，如表1所示。

\section{表1 两组患者治疗总有效率比较 $[\boldsymbol{n}(\%)]$}

\begin{tabular}{cccccc}
\hline 组别 & 例数 & 显效 & 有效 & 无效 & 总有效率 \\
\hline 对照组 & 45 & $16(35.56)$ & $21(46.67)$ & $8(17.78)$ & $37(82.22)$ \\
观察组 & 45 & $27(60.00)$ & $16(35.56)$ & $2(4.44)$ & $43(95.56)$ \\
$\chi^{2}$ & & & & & 7.645 \\
$P$ & & & & & 0.006 \\
\hline
\end{tabular}

（二）比较两组各项临床指标情况

观察组起效时间、住院时间以及治疗费用等各项治疗指标均优于对照组, 差异具有统计学意义 $(P<0.05)$, 如 表2所示。

表2 两组各项临床指标情况比较 $\left(\bar{x}_{ \pm} \boldsymbol{s}\right)$

\begin{tabular}{cccc}
\hline 组别 & 起效时间 $(\mathrm{h})$ & 住院时间 $(\mathrm{d})$ & 治疗费用 $($ 万元 $)$ \\
\hline 对照组 $(n=45)$ & $12.35 \pm 5.68$ & $33.76 \pm 5.24$ & $2.63 \pm 0.42$ \\
观察组 $(n=45)$ & $6.83 \pm 3.21$ & $26.58 \pm 3.89$ & $1.86 \pm 0.51$ \\
$t$ & 5.521 & 7.615 & 9.726 \\
$P$ & 0.000 & 0.001 & 0.001 \\
\hline
\end{tabular}


（三）比较两组治疗前后生活自理能力评分

两组患者经过治疗后生活能力评分有显著提高, 且治疗后观察组生活能力评分显著高于对照组, 差异具有统计学 意义 $(P<0.05)$ ，如表3所示。

表3 两组治疗前后生活自理能力评分比较 $(\bar{x} \pm s$, 分 $)$

\begin{tabular}{ccccc}
\hline 组别 & 例数 & 治疗前 & 治疗后 2 周 & 治疗后 $28 \mathrm{~d}$ \\
\hline 对照组 & 45 & $47.23 \pm 6.52$ & $57.24 \pm 6.12$ & $72.96 \pm 6.82$ \\
观察组 & 45 & $46.71 \pm 5.89$ & $66.48 \pm 5.81$ & $83.21 \pm 6.43$ \\
$t$ & & 0.382 & 7.396 & 7.326 \\
$P$ & & 0.706 & 0.000 & 0.000 \\
\hline
\end{tabular}

(四) 比较两组治疗后不良反应发生情况

两组不良反应发生率分别为 $4.44 \%$ 与 $6.67 \% ，$ 观察组与对照组无明显差异 $(P>0.05) ，$ 如表4所示。

表4 两组治疗后不良反应发生情况比较 $[\boldsymbol{n}(\%)]$

\begin{tabular}{ccccccc}
\hline 组别 & 例数 & 恶心 & 发热 & 头痛 & 面部潮红 & 总发生率 \\
\hline 对照组 & 45 & $1(2.22)$ & $0(0.00)$ & $1(2.22)$ & $1(2.22)$ & $3(6.67)$ \\
观察组 & 45 & $0(0.00)$ & $0(0.00)$ & $1(2.22)$ & $1(2.22)$ & $2(4.44)$ \\
$\chi^{2}$ & & & & & 0.695 \\
$P$ & & & & & 0.406 \\
\hline
\end{tabular}

\section{四、讨论}

急性格林巴利综合征是一种自身免疫系统疾病, 病理改变主要表现为周围神经组织小血管淋巴细胞浸润、神经纤 维退髓鞘, 其发病机理目前上不明确, 临床上仅能够确认其与免疫因素相关 ${ }^{[12-13]}$ 。格林巴利综合征大部分患者发病前 数日内会存在感染史, 包括发热、呕吐、腹泻、喉咙疼痛等, 而急性格林巴利综合征起病多表现为急骤, 且短时间内 病情发展迅速, 一般在1 2周内会达到高峰 ${ }^{[14]}$ 。患者发病后有较大的可能会对脑神经造成损伤, 并且还有较大概率会 出现呼吸机肌麻㾇以及球麻痹等问题，患者多表现为四肢肌肉无力，伴有不同程度的神经功能障碍，严重可因呼吸、 吞咽功能受损造成自主呼吸麻痹以及吞药困难, 存在较大致残风险, 必须积极采取措施治疗, 避免疾病继续发展, 提 高患者生存质量。

就临床研究结果显示, 男女均可发病, 且以青壮年男性发病率高。因为并未确认发病机制, 目前临床上缺乏治疗 急性格林巴利综合征的特异性手段, 单一药物治疗效果欠佳。目前临床上治疗急性格林巴利综合征的方法中多数会选 择应用免疫球蛋白疗法及激素疗法, 另外可选择应用血浆置换法以及免疫抑制疗法等, 综合分析患者病历资料与实际 情况择优选择。

因为急性格林巴利综合征与机体自身免疫机制密切相关, 临床上治疗主要从调节免疫功能方面着手, 免疫球蛋白 和甲波尼龙是最为常见的两种药物 ${ }^{[15]}$ 。两种药物的作用机理不同, 选择应用免疫球蛋白治疗, 是利用其外源性补充免 疫球蛋白的特点, 患者在接受给药治疗后, 可以促使机体重新建立免疫保护状态, 进而实现对患者机体免疫功能的改 善目的，有效预防疾病的继续发展并且，还能够为患者提供特异性抗体，成功阻断吞噬细胞 Fc受体，预防炎症的发 生，具有良好的抗炎效果。而甲波尼龙作为中效糖皮质激素，对患者进行给药治疗的目的在于对患者机体代谢功能进 行调节改善，抑制免疫应答，局部抗炎效果显著，并且药效可以保持 $12 \sim 36 \mathrm{~h}$ 。通过甲泼尼龙给药治疗后，患者的脱 髓鞘病变组织水肿症状会得到有效改善, 对神经传导阻滞可以进行逆转, 加速髓鞘的修复, 促使髓鞘区域神经传导功 能恢复。免疫球蛋白联合甲泼尼龙治疗急性格林巴利综合征, 能够进一步提高临床疗效。就本次研究结果来看, 联 合治疗总有效率更高，观察组与对照组分别为 $95.56 \%$ 和 $82.22 \%$, 观察组显著优于对照组，差异具有统计学意义 $(P<$ $0.05)$; 并且, 观察组起效时间、住院时间以及治疗费用等各项治疗指标均优于对照组, 以及两组患者经过治疗后生 活能力评分有显著提高, 且治疗后两组生活能力评分有显著差异, 观察组优于对照组 $(P<0.05)$ 。相比单纯应用免 疫球蛋白治疗，联合治疗方案患者不良反应发生率比较无明显差异，两组分别为 $4.44 \%$ 与 $6.67 \%(P>0.05)$, 具有较 高安全性。

综上所述，免疫球蛋白联合甲泼尼龙治疗急性格林巴利综合征临床效果显著，相比单纯应用免疫球蛋白治疗联合疗 
法临床总有效率更高, 可应用更短时间改善患者各临床症状, 提高患者生活自理能力, 提高其生存质量。并且, 免疫球 蛋白联合甲泼尼龙疗法花费更少，减轻患者心理以及经济负担，更利于其康复，因此在临床上值得进一步推广应用。

\section{参考文献:}

[1]刘博.免疫球蛋白联合甲泼尼龙治疗急性格林巴利综合征的疗效观察[J].现代诊断与治疗, 2019,30(10):1613-1614.

[2]张洁.免疫球蛋白、甲泼尼龙、鼠神经生长因子联合治疗慢性格林-巴利综合征患者的疗效观察 [J].中国民康医学, 2019,31(05):25-27.

[3]李艳.对格林巴利综合征治疗中常规方法与免疫球蛋白的使用效果进行对比探讨[J].中外医疗, 2019,38(07):16-18.

[4]刘荣.大剂量免疫球蛋白联合甲泼尼龙治疗急性格林巴利综合征的疗效观察[J]. 当代医学, 2018,24(26):44-46.

[5]王娜.丙种球蛋白联合激素治疗急性格林巴利综合征的效果评价[J].当代医药论丛, 2018,16(14):79-80.

[6]姜颖,赵欢.甲泼尼龙联合丙种球蛋白治疗急性格林巴利综合征的临床效果[J]. 实用临床医药杂志, 2017,21(09):179180 .

[7]穆杭.免疫球蛋白联合甲泼尼龙治疗急性格林巴利综合征的可行性观察[J]. 中外医学研究, 2017,15(04):34-35.

[8]张亚琼.激素结合免疫球蛋白治疗格林巴利综合征患者的疗效研究 [J].世界最新医学信息文摘, 2016,16(94):80.

[9]张翼,杜鍂.不同剂量免疫球蛋白联合激素治疗急性格林巴利综合征患者的效果比较[J]. 医疗装备, 2016,29(18):132.

[10]云孝慧.免疫球蛋白联合甲泼尼龙治疗急性格林巴利综合征的临床分析[J].中国实用医药, 2016,11(19):167-168.

[11]段金平.丙种球蛋白联合甲泼尼龙冲击治疗急性格林-巴利综合征的疗效观察 [J].中国医院用药评价与分析, 2016,16(04):458-460.

[12]代秀峰.免疫球蛋白联合甲泼尼龙治疗急性格林巴利综合征的临床分析[J].世界最新医学信息文摘, 2016,16(20):7879.

[13]宋晓红.免疫球蛋白联合甲泼尼龙治疗急性格林巴利综合征的可行性探讨[J].中国继续医学教育, 2015,7(24):148149.

[14]金飞锋,王海燕, 宋佳贤, 李毅鸣.免疫球蛋白联合甲泼尼龙治疗重型-极重型格林巴利综合征效果观察[J].中国乡村 医药, 2012,19(14):30-31.

[15]周劲松.大剂量免疫球蛋白联合甲泼尼龙治疗急性格林巴利综合征的临床疗效[J].中国现代药物应用, 2012,6(02):1-2. 